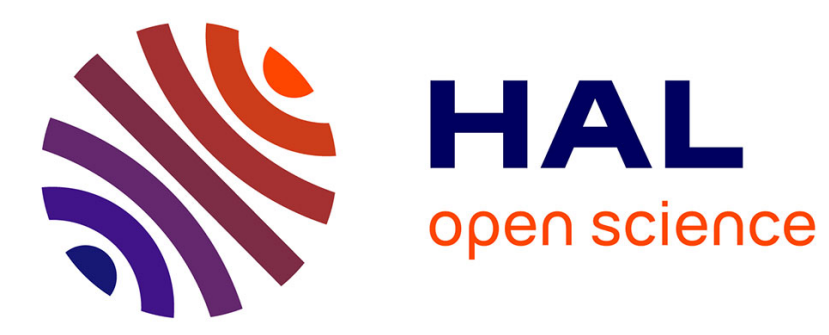

\title{
Physical Models and Control of the Train Dynamics in a Metro Line Without Junction
}

\author{
Nadir Farhi
}

\section{To cite this version:}

Nadir Farhi. Physical Models and Control of the Train Dynamics in a Metro Line Without Junction. IEEE Transactions on Control Systems Technology, 2018, 9p. 10.1109/TCST.2018.2830305 . hal01871931

\section{HAL Id: hal-01871931 \\ https://hal.science/hal-01871931}

Submitted on 11 Sep 2018

HAL is a multi-disciplinary open access archive for the deposit and dissemination of scientific research documents, whether they are published or not. The documents may come from teaching and research institutions in France or abroad, or from public or private research centers.
L'archive ouverte pluridisciplinaire HAL, est destinée au dépôt et à la diffusion de documents scientifiques de niveau recherche, publiés ou non, émanant des établissements d'enseignement et de recherche français ou étrangers, des laboratoires publics ou privés. 


\title{
Physical Models and Control of the Train Dynamics in a Metro Line Without Junction
}

\author{
Nadir Farhi ${ }^{(\infty)}$
}

\begin{abstract}
We propose a traffic flow and control model for the train dynamics in a linear metro line without junction. The model takes into account time constraints such as minimum interstation running times, minimum train dwell times at platforms, and minimum safe separation times between successive trains. Moreover, it includes a control law that sets the train dwell times at platforms based on the feedback of the train time-headways and of the passenger arrival rates at platforms. We show that the dynamic system converges to a stable stationary regime with a unique average growth rate, and derive, by numerical simulation, the traffic phases of the train dynamics. We compare the obtained traffic phases with the ones derived with an existing max-plus algebra traffic model, and derive the effect of the passenger travel demand on the train dynamics. Finally, we draw some conclusions and discuss perspectives of the proposed approach.
\end{abstract}

Index Terms-Physics of traffic, railway traffic, traffic control, traffic modeling.

\section{INTRODUCTION}

C OLLECTIVE transport by metro is one of the highcapacity passenger transport systems in urban areas. Often grade separated, and operating on an exclusive rightof-way, metro systems allow the realization of high train frequencies. However, as well known, natural instability appears when a metro line is exploited at high frequencies, where the capacity margins are reduced (see [25]). Indeed, the reduction of the capacity margins amplifies train delays and eases their propagation in space and in time.

Automatic train regulation (ATR) can maintain the schedule and headway adherence against disturbances caused by minor delays (less than 3 min) [19]. The main control variables of ATR are the train running and dwell times. In fixed block systems, the preallocation of buffer slack or supplement in the time headway permits to guarantee system reliability by absorbing primary and associated secondary delays, even though this may cause a loss in capacity utilization. In moving block systems, system-wide responsive traffic regulators can substantially improve capacity and recovery capability from the local delay. The main performance indicators of the traffic in a metro line are capacity utilization, reliability, and punctuality. An important parameter that affects these indicators is the traffic stability, in particular, the robustness against disturbances.

Manuscript received January 15, 2018; revised April 17, 2018; accepted April 18, 2018. Manuscript received in final form April 23, 2018. Recommended by Associate Editor M. Dotoli.

The author is with Université Paris-Est, Ifsttar/Cosys/Grettia, F-77447 Marne-la-Vallée cedex 2, France (e-mail: nadir.farhi@ifsttar.fr).

Color versions of one or more of the figures in this paper are available online at http://ieeexplore.ieee.org.

Digital Object Identifier 10.1109/TCST.2018.2830305
Here, we propose a real-time traffic control model, where we assume that the trains follow predefined speed profiles without the possibility of control of the their interstation running times. The main control variable we consider here is the train dwell times at platforms, which are submitted to constraints on the passenger boarding and alighting times. The relationship between the train dynamics and the passenger ones is mutual. Indeed, high levels of passenger densities on platforms and/or in trains incite train dwell times to increase, while delayed trains cause the accumulation of passengers at platforms. This cyclic dependence favors amplification and propagation of traffic disturbances in time and in space.

Numerous approaches of real-time control have been adopted for railway traffic management, including optimization, simulation, expert system, and so on. An overview of recovery models and algorithms for real-time railway disturbance and disruption management can be found for example in [2].

The traffic in metro lines, including the train as well as the passenger flow dynamics, is not yet well understood. Train dynamics, passenger flowing, driver behavior, and many decision levels are combined in the transport system. While the train dynamics can be described with discrete event systems, the passenger flows are rather modeled with continuous processes, including a source of uncertainty. In nonautomated metro lines, other sources of uncertainty are due to the train driver decisions. The decision levels are many, including tactical, operational, and strategic ones. Tactical level decisions include train departure and dwell times, train speed profiles, and so on. Operational level decisions include mainly the optimization of train time tables. The strategic level decisions include all the higher level ones taking into account the passenger demand, up to planning decisions.

Due to the complexity of the traffic dynamics in metro line systems, most of the traffic control approaches are based on optimization and operations research. We cite below some of the existing works in this direction. Cury et al. [4] proposed an analytical traffic model with a multilevel hierarchical optimization method. Goodman and Murata [17] used nonlinear optimization with constraints, where the objective function takes into account passenger flows. The authors investigated the optimization approaches for metro traffic regulation, which focus on the customer perceptions of the service quality. The approach consists in penalizing excess waiting and travel times, as well as congestion. It is illustrated by means of data based on the Hong Kong Island line, and is compared to existing control algorithms. Assis and Milani [1] solved the 
train scheduling problem in metro lines by a predictive control method. Engelhardt-Funke and Kolonko [5] have proposed a stochastic model for the propagation of the train delays across the network, where the waiting time for passengers changing lines is minimized. Sun et al. [24] developed a mathematical programming approach to minimize waiting time, where the Lagrangian duality theory is used to solve the problem. Train schedules that take into account passenger exchanges on platforms have been generated.

One of the first traffic models that point out the nature of traffic instability in metro lines is due to Van Breusegem et al. [25]. Van Breusegem et al. [25] proposed a linear quadratic (LQ) control approach to deal with the instability. The approach is based on the discrete-event traffic and control models. Control algorithms assuring system stability are proposed and the approach has been illustrated by simulations. Since then, several improvements have been proposed to the model. Lin and Shu [20] basing on the model of Van Breusegem et al. [25], proposed an algorithm that approximates an LQ regulator by learning traffic data with artificial neural networks. The algorithm is based on a critic-actor architecture of reinforcement learning. The main advantage of this approach is that the traffic regulator is not biased by the traffic modeling errors. The authors illustrate the approach and verify it in a simulated system with traffic data acquired from the Taipei Metro System. Recently, Schanzenbächer et al. [21] applied the approach of [25] on a stretch of the RER A line of Paris.

Although we are interested in traffic control and optimization, we here adopt the approach of Farhi et al. [12]-[14], which permits the understanding of the physics of traffic in a metro line, and in particular the effect of the passenger demand on the traffic phases of the train dynamics. Fundamental traffic diagrams similar to the ones derived in the road traffic (see [6]-[11]) are derived in [12]-[14]. As mentioned earlier, we propose a control law for the train dwell times as functions of the passenger arrival rates onto the platforms, and derive the effect of the passenger demand on the physics of traffic.

The outline of this brief is as follows. In Section II, we give a short review on the dynamic programming systems. In Section III, we present the model of the train dynamics with a traffic control law that sets the train dwell times on the feedback of both the train time-headway and the passenger arrivals. The model guarantees the stability of the train dynamics, interpreted, in this case, as a dynamic programming system. The traffic phase diagrams for the train dynamics are derived by simulation. The effect of the passenger arrivals on the traffic phases is shown. Finally, conclusions and perspectives are given in Section IV.

\section{Short Review On DyNAMiC PROGRAMMING SYSTEMS}

The traffic models we discuss in this brief have the form of dynamic programming systems, for which we provide a short review in this section.

A map $\mathbf{f}: \mathbb{R}^{n} \rightarrow \mathbb{R}^{n}$ is said to be additive 1-homogeneous if it satisfies: $\forall x \in \mathbb{R}^{n}, \forall a \in \mathbb{R}, \mathbf{f}(a \mathbf{1}+x)=a \mathbf{1}+\mathbf{f}(x)$, where
$1 \stackrel{\text { def }}{=}{ }^{t}(1,1, \ldots, 1)$. It is said to be monotone if it satisfies $\forall x, y \in \mathbb{R}^{n}, x \leq y \Rightarrow \mathbf{f}(x) \leq \mathbf{f}(y)$, where $x \leq y$ means $x_{i} \leq$ $y_{i} \forall i, 1 \leq i \leq n$. If $\mathbf{f}$ is 1 -homogeneous and monotone, then it is nonexpansive (or 1-Lipschitz) for the supremum norm [3], i.e., $\forall x, y \in \mathbb{R}^{n},\|\mathbf{f}(x)-\mathbf{f}(y)\|_{\infty} \leq\|x-y\|_{\infty}$. In this case, a directed graph $\mathcal{G}(\mathbf{f})$ is associated with $\mathbf{f}$.

The directed graph $\mathcal{G}(\mathbf{f})$ associated with $\mathbf{f}: \mathbb{R}^{n} \rightarrow \mathbb{R}^{n}[16]$ is defined by the set of nodes $\{1,2, \ldots, n\}$ and by a set of arcs such that there exists an arc from a node $i$ to a node $j$ if $\lim _{\eta \rightarrow \infty} \mathbf{f}_{i}\left(\eta e_{j}\right)=\infty$, where $e_{j}$ is the $j$ th vector of the canonical basis of $\mathbb{R}^{n}$. We review below an important result on the existence of additive eigenvalues of 1-homogeneous monotone maps.

Theorem 1 [15], [18]: If $\mathbf{f}: \mathbb{R}^{n} \rightarrow \mathbb{R}^{n}$ is 1 -homogeneous and monotone and if $\mathcal{G}(\mathbf{f})$ is strongly connected then $\mathbf{f}$ admits an (additive) eigenvalue, i.e., $\exists \mu \in \mathbb{R}, \exists x \in \mathbb{R}^{n}: \mathbf{f}(x)=$ $\mu+x$. Moreover, we have $\chi(\mathbf{f})=\mu \mathbf{1}$, where $\chi(\mathbf{f})$ denotes the asymptotic average growth rates vector of the dynamic system $x(k+1)=\mathbf{f}(x(k))$, defined by: $\chi(\mathbf{f})=\lim _{k \rightarrow \infty} \mathbf{f}^{k}(x) / k$.

For simplicity, we use in this brief, for all the dynamic systems, the notation $x^{k}$ instead of $x(k)$. We give in the following a natural extension of Theorem 1, which will permit us to consider dynamic systems of the form $x^{k}=$ $\mathbf{f}\left(x^{k}, x^{k-1}, \ldots, x^{k-m+1}\right)$. For that, let us consider $\mathbf{f}: \mathbb{R}^{m \times n} \rightarrow$ $\mathbb{R}^{n}$ associating for $\left(x^{0}, x^{1}, \ldots, x^{m-1}\right)$, where $x^{i}$ are vectors in $\mathbb{R}^{n}$, a column vector in $\mathbb{R}^{n}$

$$
\begin{aligned}
\mathbf{f}: \mathbb{R}^{m \times n} & \rightarrow \mathbb{R}^{n} \\
\left(x^{0}, x^{1}, \ldots, x^{m-1}\right) & \mapsto \mathbf{f}(x) .
\end{aligned}
$$

We denote by $\mathbf{f}_{i}, i=0,1, \ldots, m-1$, the following maps:

$$
\begin{aligned}
\mathbf{f}_{i}: \mathbb{R}^{n} & \rightarrow \mathbb{R}^{n} \\
x & \mapsto \mathbf{f}_{i}(x)=\mathbf{f}(-\infty, \ldots,-\infty, x,-\infty, \ldots,-\infty) \\
\uparrow & \begin{array}{c}
\uparrow \\
i^{\text {th }} \text { component }
\end{array}
\end{aligned}
$$

and by $\tilde{\mathbf{f}}$, the following map:

$$
\begin{aligned}
\tilde{\mathbf{f}}: \mathbb{R}^{n} & \rightarrow \mathbb{R}^{n} \\
x & \mapsto \tilde{\mathbf{f}}(x)=\mathbf{f}(x, x, \ldots, x) .
\end{aligned}
$$

Theorem 2: If $\tilde{\mathbf{f}}$ is additive 1-homogeneous and monotone, and if $\mathcal{G}(\tilde{\mathbf{f}})$ is strongly connected and $\mathcal{G}\left(\mathbf{f}_{0}\right)$ is acyclic, then f admits a unique generalized additive eigenvalue $\mu>-\infty$ and an additive eigenvector $v>-\infty$, such that $\mathbf{f}(v, v-\mu$, $v-2 \mu, \ldots, v-(m-1) \mu)=v$. Moreover, $\chi(\mathbf{f})=\mu \mathbf{1}$.

Proof: The proof consists in showing that the dynamic system $x^{k}=\mathbf{f}\left(x^{k}, x^{k-1}, \ldots, x^{k-m+1}\right)$ is equivalent to another dynamic system $z^{k}=\mathbf{h}\left(z^{k-1}\right)$, where $\mathbf{h}$ is built from $\mathbf{f}$, such that $\mathbf{h}$ satisfies additive 1-homogeneity, monotonicity, and connectivity properties needed by Theorem 1 . The whole proof is available in [12].

\section{TRAFFiC MOdel}

Here, we propose a traffic model for the train dynamics in a metro line. The model is based on the max-plus linear model of [13]. More precisely, it extends it in order to take into account the passenger arrivals and their effects on the 


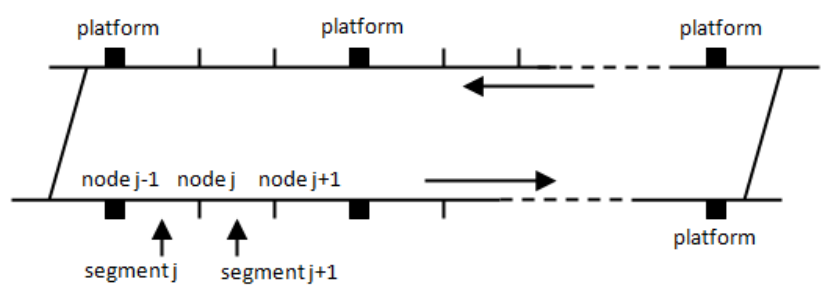

Fig. 1. Representation of a linear metro line.

train dwell times and on the train dynamics, in general. We notice that the model presented in [14] proposed such an extension, but with an assumption of unlimited train capacity in terms of the maximum number of passengers. The model we present here improves the one of [14] by considering passenger capacity limits of the trains, which is more realistic. This new modeling modifies consequently the traffic control law as well as the derived traffic phases.

The model writes the train departure times under two constraints on the interstation travel time (running time + dwell time), and on the safety time between successive trains. Moreover, it describes the train dynamics by applying a control of the train dwell times on platforms, based on the feedback of the passenger travel demand. We do not consider, in this brief, a dynamic model for the number of passengers at platforms. Therefore, we assume that the dwell times at platforms depend directly on the passenger arrival rates.

Let us consider a linear metro line of $N$ platforms without junction, as shown in Fig. 1. In order to model the train dynamics on the whole line, including the dynamics on interstations, we discretize the interstation spaces, and thus the whole line, on segments (or sections or blocks). The length of every segment must be larger than the length of a train. In case of a fixed block signaling system, the discretization in segments we make here reflects the fixed blocks. In case of a moving block system, our discretization permits the approximation of the train dynamics. Let us consider the following notations.

$n \quad$ Number of all segments of the line.

$m \quad$ Number of running trains.

$L \quad$ Length of the whole line.

$b_{j} \in\{0,1\}$ : boolean number of trains being on segment $j$ at time zero.

$\bar{b}_{j} \quad 1-b_{j} \in\{0,1\}$.

$d_{j}^{k} \quad$ Instant of the $k$ th departure from node $j$. Notice that $k$ do not index trains, but count the number of departures from segment $j$.

$a_{j}^{k} \quad$ Instant of the $k$ th arrival to node $j$.

$r_{j} \quad$ The running time of a train on segment $j$, i.e., from node $j-1$ to node $j$.

$w_{j}^{k} \quad d_{j}^{k}-a_{j}^{k}$ : train dwell time corresponding to the $k$ th arrival to- and departure from node $j$.

$t_{j}^{k} \quad r_{j}+w_{j}^{k}$ : train travel time from node $j-1$ to node $j$, corresponding to the $k$ th arrival to- and departure from node $j$.

$g_{j}^{k} \quad a_{j}^{k}-d_{j}^{k-1}$ : node- (or station-) safe separation time (also known as close-in time), corresponding to the $k$ th arrival to- and $(k-1)$ st departure from node $j$. $h_{j}^{k} \quad d_{j}^{k}-d_{j}^{k-1}=g_{j}^{k}+w_{j}^{k}:$ departure time headway at node $j$, associated with the $(k-1)$ st and $k$ th departures from node $j$.

$s_{j}^{k} \quad g_{j}^{k+b_{j}}-r_{j}^{k}$ : a kind of node safe separation time which does not take into account the running time.

We also use underlined and overlined notations to denote the maximum and minimum bounds of the corresponding variables, respectively. Then, $\bar{r}_{j}, \bar{t}_{j}, \bar{w}_{j}, \bar{g}, \bar{h}_{j}$, and $\bar{s}_{j}$ and, respectively, $\underline{r}_{j}, \underline{t}_{j}, \underline{w}_{j}, \underline{g}, \underline{h}_{j}$, and $\underline{s}_{j}$ denote the maximum and minimum running, travel, dwell, safe separation, headway, and $s$ times, respectively.

The average on $j$ and on $k$ (asymptotic) of those variables are denoted without any subscript or superscript. Then $r, t, w, g, h$, and $s$ denote the average running, travel, dwell, safe separation, headway, and $s$ times, respectively.

It is easy to check the following relationships:

$$
\begin{aligned}
g & =r+s \\
t & =r+w \\
h & =g+w=t+s=\frac{n}{m} t=\frac{n}{n-m} s .
\end{aligned}
$$

Indeed, (1) comes from the definition of $s_{j}^{k}$ and (2) comes from the definition of $t_{j}^{k}$. For (3):

1) $h=g+w$ comes from the definition of $h_{j}^{k}$;

2) $h=t+s$ comes from the definition of $t_{j}^{k}$ and $s_{j}^{k}$ and from $h=g+w$;

3) $h=n t / m$ average train time-headway is given by the travel time of the whole line $(n t)$ divided by the number of trains;

4) $h=n s /(n-m)$ can be derived from $h=t+s$ and $h=n t / m$.

The running times $r_{j}$ of trains on every segment $j$ are assumed to be constant. They can be calculated from given interstation speed profiles, depending on the characteristics of the line and of the trains running on it. We then have $\underline{t}_{j}=r_{j}+\underline{w}_{j}$ and $\bar{t}_{j}=r_{j}+\bar{w}_{j}$ for every $j$.

An important remark here is that the variables $w_{j}^{k}$ denote dwell times at all the nodes $j \in\{1, \ldots, n\}$ including nonplatform nodes. The lower bounds $\underline{w}_{j}$ should be zero for the nonplatform nodes $j$, and they should be strictly positive for platform nodes (we assume that all the trains stop at all the platforms). Therefore, the asymptotic average dwell time $w$ at all the nodes is different from (it is lower than) the asymptotic average dwell time at platform nodes, which we denote $w^{*}$.

In our model, we consider constant running times on segments and on interstations. Therefore, every train deceleration or stop at the level of an interstation, generally caused by an interaction with the train ahead, is modeled here by a dwell time extension at one of the nodes of the considered interstation. The interstation train running times can also be considered as control variables, in addition to train dwell times at platforms. In such modeling, shortened interstation train running times can compensate extended train dwell times at platforms, while extended running times can be considered for eco-driving and optimization of energy consumption. We shall consider this extended modeling in our future research. 
The model is built on the three time constraints as follows. 1) A constraint on the travel time on every segment $j$

$$
d_{j}^{k} \geq d_{j-1}^{k-b_{j}}+\underline{t}_{j}
$$

Constraint (4) tells us first that the $k$ th departure from node $j$ corresponds to the $k$ th departure from node $(j-1)$ in case where there is no train at segment $j$ at time zero $\left(b_{j}=0\right)$, and corresponds to the $(k-1)$ st departure from node $(j-1)$ in case where there is a train at segment $j$ at time zero. Constraint (4) tells us in addition that the departure from node $j$ cannot be realized before the corresponding departure from node $(j-1)$ plus the minimum travel (run + dwell) time from node $j-1$ to node $j$.

2) A constraint on the safe separation time at every segment $j$

$$
\begin{aligned}
d_{j}^{k}-d_{j+1}^{k-\bar{b}_{j+1}} & =a_{j+1}^{k+b_{j+1}}-r_{j+1}-d_{j+1}^{k-\bar{b}_{j+1}} \\
& =g_{j+1}^{k+b_{j+1}}-r_{j+1} \\
& \geq \underline{g}_{j+1}-r_{j+1}=\underline{s}_{j+1} .
\end{aligned}
$$

That is,

$$
d_{j}^{k} \geq d_{j+1}^{k-\bar{b}_{j+1}}+\underline{s}_{j+1} .
$$

Constraint (5) tells us first that, in term of safety, the $k$ th departure from node $j$ is constrained by the $(k-1)$ st departure from node $(j+1)$ in case where there is no train at segment $(j+1)$ at time zero, and it is constrained by the $k$ th departure from node $(j+1)$ in case where there is a train at segment $(j+1)$ at time zero. Constraint (5) tells us in addition that the $k$ th departure from node $j$ cannot be realized before the departure constraining it from node $(j+1)$ plus the minimum safety time at node $(j+1)$.

3) A constraint on the train dwell time at every platform taking into account the passenger arrivals

$$
w_{j}^{k} \geq\left\{\begin{array}{l}
\bar{w}_{j}-\frac{\theta_{j}^{k}\left(\alpha_{j}^{k}-\lambda_{j}^{k}\right)}{\lambda_{j}^{k}} g_{j}^{k} \\
\text { if } j \text { indexes a platform } \\
\text { otherwise }
\end{array}\right.
$$

where $\alpha_{j}$ is the average passenger upload rate from platform $j$ onto the trains (during train dwell time), if $j$ indexes a platform; and $\alpha_{j}$ is zero otherwise; and $\lambda_{j}$ is the average rate of the total arrival flow of passengers to platform $j$, if $j$ indexes a platform; and $\lambda_{j}$ is zero otherwise

$$
\lambda_{j}= \begin{cases}\sum_{i} \lambda_{j i} & \text { if } j \text { indexes a platform } \\ 0 & \text { otherwise }\end{cases}
$$

where $\lambda_{j i}$ denote here the origin-destination arrival rates of passengers to platform $j$, with platform $i$ as destination. $\bar{w}_{j}$ (maximum dwell time on node $j$ ) and $\theta_{j}^{k}$ are control parameters to be fixed.
Let us notice that the control law (6) is equivalent to

$$
w_{j}^{k} \geq \begin{cases}\bar{w}_{j}-\frac{\theta_{j}^{k} \alpha_{j}^{k}}{\lambda_{j}^{k}} h_{j}^{k} & \text { if } j \text { indexes a platform } \\ 0 & \text { otherwise }\end{cases}
$$

which is implicit since $h_{j}^{k}=g_{j}^{k}+w_{j}^{k}$. We also notice that we have in general $\alpha_{j}-\lambda_{j}>0$, otherwise, the passenger flows can never be served.

It has already been noticed in [12], [13], and [25] that the train dwell times tend to have the natural (uncontrolled) behavior

$$
w_{j}^{k} \geq\left(\lambda_{j}^{k} / \alpha_{j}^{k}\right) h_{j}^{k} \quad \text { if } j \text { indexes a platform }
$$

where only the effect of the boarding time of passengers into the trains, on the train dwell time, is considered, without considering the alighting time effect. The behavior (8) causes instability of the train dynamics [12], [13], [25]. In (7), we reversed [with respect to (8)], the sign of the relationship between the dwell time $w_{j}^{k}$ and the train time headway $h_{j}^{k}$, without reversing the relationship between the dwell time $w_{j}^{k}$ and the ratio $\lambda_{j}^{k} / \alpha_{j}^{k}$.

Combining the constraints (4)-(6), the train dynamics are written, for nodes $j$ indexing platforms, as follows:

$$
d_{j}^{k}=\max \left\{\begin{array}{l}
d_{j-1}^{k-b_{j}}+r_{j}+\underline{w}_{j} \\
\left(1-\delta_{j}^{k}\right) d_{j-1}^{k-b_{j}}+\delta_{j}^{k} d_{j}^{k-1}+\left(1-\delta_{j}^{k}\right) r_{j}+\bar{w}_{j} \\
d_{j+1}^{k-\bar{b}_{j+1}}+\underline{s}_{j+1}
\end{array}\right.
$$

where $\delta_{j}^{k}=\theta_{j}^{k}\left(\alpha_{j}^{k}-\lambda_{j}^{k}\right) / \lambda_{j}^{k}, \forall j, k$ and the index $j$ is taken with modulo $n$. For nonplatform nodes, the dynamics combines only the constraints (4) and (5). Here, we recall that the maxplus algebra model of [13] is obtained by the combination of (only) two constraints (4) and (5) for all the nodes (platform and nonplatform nodes)

$$
d_{j}^{k}=\max \left\{d_{j-1}^{k-b_{j}}+\underline{t}_{j}, d_{j+1}^{k-\bar{b}_{j+1}}+\underline{s}_{j+1}\right\} . \forall j .
$$

If $\delta_{j}^{k}$ are independent of $k$ for every $j$, then the dynamic system (9) can be written in the following form:

$$
d_{j}^{k}=\max _{u \in \mathcal{U}}\left[\left(M^{u} d^{k-1}\right)_{j}+\left(N^{u} d^{k}\right)_{j}+c_{j}^{u}\right]
$$

where $M^{u}$ and $N^{u}$ are square matrices and $c^{u}$ are column vectors, for $u \in \mathcal{U}$. The matrices $N^{u}, u \in \mathcal{U}$ express implicit terms. If $m=0$ or $m=n$, then the system (9) is fully implicit, and admits an asymptotic regime with a unique asymptotic average growth rates vector, interpreted here as the train timeheadways at the nodes $h_{j}=+\infty, \forall j$ (no train movement is possible). If $0<m<n$, then the dynamic system (9) is triangular. In this case, and if $\delta_{j}^{k}$ are independent of $k$ for every $j$, and $0 \leq \delta_{j} \leq 1, \forall j$, then for the dynamic system (9), written under the form (11), $M^{u}$ and $N^{u}$ are substochastic matrices, i.e., satisfying $M_{i j}^{u} \geq 0, N_{i j}^{u} \geq 0, \sum_{j}\left(M_{i j}^{u}\right) \leq 1$, and $\sum_{j}\left(N_{i j}^{u}\right) \leq 1$. Moreover, we have $\sum_{j}\left(M_{i j}^{u}+N_{i j}^{u}\right)=1, \forall i, u$. In this case, (9) is a dynamic programming system of an optimal control problem of a Markov chain, whose transition matrices can be calculated from $M^{u}, u \in \mathcal{U}$ and $N^{u}, u \in \mathcal{U}$ [they are the matrices corresponding to the equivalent explicit 
dynamic system obtained by solving the implicit terms of (9)]; and whose reward vectors are $c^{u}, u \in \mathcal{U}$. Here, we assume that $\delta_{j}^{k}$ are independent of $k$, which means practically that $\lambda_{j}^{k}$ are time-independent. Therefore, the model is to be applied separately on each time-period of stable passenger arrival rates.

Theorem 3: If $\delta_{j}^{k}$ are independent of $k$ for every $j$, and if $0 \leq \delta_{j} \leq 1, \forall j$, then the dynamic system (9) admits a stationary regime with a unique additive eigenvalue $h$, which coincides with all the components of the asymptotic average growth rates vector of the system, independent of the initial state vector $d^{0}$.

Proof: The proof uses Theorem 2 (see [12]).

We do not yet have an analytic formula for the asymptotic train time-headway (which we know that it coincides with eigenvalue $h$ ), but Theorem 3 guarantees its existence and its uniqueness. Therefore, by iterating the dynamics (9), one can approximate, for any fixed train density $\rho$, the associated asymptotic average train time-headway $h(\rho)$ as follows:

$$
h(\rho) \approx d_{j}^{K} / K \quad \forall j, \text { for a large } K .
$$

Let us define the asymptotic variances (in time) $\operatorname{Var}_{j}$ of the growth rates of the system at each node $j$, as follows:

$$
\operatorname{Var}_{j}:=\lim _{T \rightarrow+\infty} \frac{1}{T} \sum_{k=1}^{T}\left(h_{j}^{k}-h\right)^{2} \forall j
$$

We know that many additive eigenvectors may be associated with the unique additive eigenvalue $h$ of the dynamic system (9). Although all the components of the average growth rates vector of the system converge to $h$, the state vector $d^{k}$, depending on the initial state vector $d^{0}$, can converge (up to an additive constant) to one of the additive eigenvectors of the system (asymptotic stability), in which case $\operatorname{Var}_{j}=0, \forall j$; or attain a periodic regime around one of the additive eigenvectors of the system (asymptotic orbital stability), in which case $\exists j, \operatorname{Var}_{j}>0$. An extension of the model in order to guarantee $\operatorname{Var}_{j}=0, \forall j$ independent of the initial state vector $d^{0}$, will be considered in our future research.

The dynamic system converges (up to an additive constant) to one (or to an orbit) of the additive eigenvectors, for every initial state. The convergence is then global. Therefore, every time-limited perturbation is resorbed, because the state vector of the system at the end of the perturbation can be considered as a new initial state, and then we know that the dynamic system converges to a stationary regime with a unique asymptotic average growth rate. In order to go further and analyze the response to repeated perturbations, we need to study the transient regime of the dynamic system, its rate of convergence, and so on. We shall treat these questions in our future works.

In the following, we give a result (Theorem 4) which tells us under which conditions on the control parameters $\bar{w}_{j}$ and $\theta_{j}$ (or equivalently $\delta_{j}$ ), the dynamic system (9) is a max-plus linear system. We will use this result in Section III-A, in order to derive a general approach for fixing the control parameters $\bar{w}_{j}$ and $\delta_{j}$ in such a way that the effect of passenger arrivals on the train dynamics will be well modeled.
Theorem 4: Let $\tilde{h}$ be the asymptotic average growth rate of the max-plus linear system (10). The dynamic programming system (9) with parameters $\bar{w}_{j}=\tilde{h}, \forall j$ and $\delta_{j}=1, \forall j$, is a max-plus linear system, whose asymptotic average growth rate (time-headway) coincides with $\tilde{h}$.

Proof: The proof is available in [12].

\section{A. How to Fix the Control Parameters $\bar{w}_{j}$ and $\delta_{j}$ ?}

Theorem 4 tells us that if we fix in (9) $\left(\bar{w}_{j}, \delta_{j}^{k}\right)=(\tilde{h}(\rho), 1)$, then, we obtain a max-plus linear dynamic system. We know that in this case, the train dynamics do not take into account the passenger demand.

We assume here that $\alpha_{j}^{k}, \lambda_{j}^{k}, \theta_{j}^{k}$ and then $\delta_{j}^{k}$ are independent of $k$, and then denote $\alpha_{j}, \lambda_{j}, \theta_{j}$ and $\delta_{j}$, respectively. Let us consider the metro line as a server of passengers. We assume that the average arrival of passengers to a platform $j$ is $\lambda_{j}$, while the average service rate at the level of a platform $j$ is $\min \left(\alpha_{j}, \kappa / h\right)$, where $\kappa$ denotes the train capacity (maximum number of passengers in a train). Under the maxplus linear model (10) for the train dynamics, the asymptotic average service rate depends on the number $m$ of trains, or equivalently on the train density $\rho$ (see [13]). It is given by $\min \left(\alpha_{j}, \kappa / \tilde{h}(\rho)\right)$. Therefore, the metro line as a server operating under the max-plus linear model is stable under the condition $\lambda_{j}<\min \left(\alpha_{j}, \kappa / \tilde{h}(\rho)\right), \forall j$. Let use the following notation:

$$
\tilde{\lambda}_{j}(\rho):=\min \left(\alpha_{j}, \kappa / \tilde{h}(\rho)\right) .
$$

The stability condition of the server means that if $\lambda_{j} \leq$ $\tilde{\lambda}_{j}(\rho), \forall j$, then the server operating under the max-plus model can serve the passengers without adapting the train dwell times to the passenger arrival rates. Basing on this remark, we propose here a way of fixing the parameters $\bar{w}_{j}$ and $\delta_{j}$ in function of the passenger arrivals, in such a way as follows.

1) If $\lambda_{j} \leq \tilde{\lambda}_{j}(\rho), \forall j$, then the dynamic system behaves as a max-plus linear one. That is, the train dwell times are not constrained by the arrival rates of passengers.

2) If $\exists j, \lambda_{j}>\tilde{\lambda}_{j}(\rho)$, then the system switches to a dynamic programming one, where the train dwell times are constrained by the arrival rates of passengers.

Let us fix the parameters $\bar{w}_{j}$ and $\delta_{j}$ as follows:

$$
\begin{aligned}
\bar{w}_{j}(\rho) & :=\tilde{h}(\rho), \forall \rho, j \\
\delta_{j}(\rho) & :=\frac{\tilde{\lambda}_{j}(\rho)}{\max \left(\lambda_{j}, \tilde{\lambda}_{j}(\rho)\right)} \quad \forall \rho, j .
\end{aligned}
$$

Fixing $\delta_{j}(\rho)$ as in (16) is equivalent to fixing $\theta_{j}(\rho)$ as follows:

$$
\theta_{j}(\rho):=\frac{\tilde{\lambda}_{j}(\rho)}{\max \left(\lambda_{j}, \tilde{\lambda}_{j}(\rho)\right)} \frac{\lambda_{j}}{\left(\alpha_{j}-\lambda_{j}\right)} \forall \rho, j
$$

where in case $\alpha_{j}=\lambda_{j}$, the term $\left(\alpha_{j}-\lambda_{j}\right)$ in (17) eliminates the one in (6) without evaluation.

We then have $0 \leq \delta_{j}(\rho) \leq 1$ by definition, and:

1) if $\lambda_{j} \leq \tilde{\lambda}_{j}(\rho), \forall j$, then $\delta_{j}(\rho)=1, \forall j$, and the dynamic system is a max-plus linear one, where the dwell times are not constrained by the arrival rates of passengers and 
2) if $\exists j, \lambda_{j}>\tilde{\lambda}_{j}(\rho)$, then $\exists j, \delta_{j}(\rho)<1$, and the system switches to a dynamic programming one where the dwell times are constrained by the arrival rates of passengers.

Let us recall that we do not have here a dynamic model for the stock of passengers at platforms. Therefore, the number of passengers who failed to get onto the trains at the $k$ th departure is not memorized for the $(k+1)$ st departure. We summarize the latter findings in the following result.

Theorem 5: For any fixed value of the train density $\rho$ on the metro line, the dynamic system (9) with parameters $\bar{w}_{j}$ and $\delta_{j}$ fixed dependent on $\rho$ as in (15) and (16), respectively, admits a stationary regime with a unique additive eigenvalue $h(\rho)$, which coincides with the asymptotic average growth rate of the system, independent of the initial state $d^{0}$. We have $h(\rho) \geq \tilde{h}(\rho)$.

Proof: The proof follows from Theorem 3 and from all the arguments given earlier in this section, in particular from $0 \leq \delta_{j}(\rho) \leq 1, \forall \rho, j$.

\section{B. How Many Trains to Retrieve the Max-Plus Dynamics}

From Theorem 4, we know that for $\delta_{j}=1, \forall j$, the max-plus dynamics are retrieved, in which case, the passenger arrivals do not have any effect on the train dynamics. From (16), we deduce that this is realized as long as $\tilde{\lambda}_{j}(\rho) \geq \lambda_{j}, \forall j$, that is under the condition

$$
\min (\alpha, \kappa / \tilde{h}(\rho)) \geq \lambda_{j} \quad \forall j
$$

or equivalently

$$
\alpha \geq \lambda_{j} \quad \forall j
$$

and

$$
\kappa / \tilde{h}(\rho) \geq \lambda_{j} \quad \forall j .
$$

Condition (18) is independent of $\rho$, then if (18) is not satisfied, then the max-plus dynamics cannot be attained for any train density $\rho$. However, condition (18) is in general largely satisfied. Indeed, as fixed in Table I below, $\alpha$ is about 30 passengers/s, while $\lambda_{j}$ exceeds rarely 10 passengers/s. Here, we assume that (18) is satisfied. Therefore, the condition such that the max-plus dynamics will be attained is (19), or equivalently $\tilde{f}(\rho) \geq \lambda_{j} / \kappa$, where $\tilde{f}(\rho)=1 / \tilde{h}(\rho)$ is the asymptotic average train frequency under the max-plus train dynamics derived in [13].

$$
\tilde{f}(\rho)=\min \left\{v \rho, f_{\max }, w^{\prime}(\bar{\rho}-\rho)\right\}
$$

where $v$ is the free (or maximum) train-speed on the metro line, $f_{\max }=1 / \max _{j}\left(\underline{t}_{j}+\underline{s}_{j}\right)$ is the train capacity (maximum train frequency), $w^{\prime}$ is the backward wave-speed for the train dynamics, and $\bar{\rho}$ is the maximum train density in the metro line (see [13]). Hence, from (20), the conditions under which the max-plus dynamics are attained, are

$$
\lambda_{j} \leq \kappa f_{\max } \quad \forall j
$$

and

$$
\frac{\lambda_{j}}{v \kappa} \leq \rho \leq \bar{\rho}-\frac{\lambda_{j}}{w^{\prime} \kappa} \quad \forall j
$$

TABLE I

PARAMETERS OF THE LINE CONSIDERED

\begin{tabular}{|l||l|}
\hline Number of stations & $9(\Rightarrow 18$ platforms $)$ \\
\hline Segment length & about 200 meters $(\mathrm{m})$. \\
\hline Free train speed $v_{\text {run }}$ & $22 \mathrm{~m} / \mathrm{s} \mathrm{(about} 80 \mathrm{~km} / \mathrm{h})$ \\
\hline Train speed on terminus & $11 \mathrm{~m} / \mathrm{s}$ (about $40 \mathrm{~km} / \mathrm{h})$ \\
\hline Min. dwell time $\underline{w}$ & 20 seconds \\
\hline Min. safety time $\underline{s}$ & 30 seconds \\
\hline Metro line length & $17.294 \mathrm{~km}$ \\
\hline Passenger train capacity $\kappa$ & 500 passenger/train \\
\hline Passenger upload rate $\alpha$ & 30 passengers $/ \mathrm{s}$ \\
\hline
\end{tabular}

Condition (21) tells us that if for any $j$, the passenger arrival rate $\lambda_{j}$ is higher than $\kappa f_{\text {max }}$, then, the max-plus train dynamics cannot be retrieved, whatever the number of trains we made in the metro line. Under the condition (21), condition (22) gives the number of trains to run on the metro line in order that the train dynamics behaves as the max-plus one. Here, we notice that the first inequality $\left[\rho \geq \lambda_{j} /(v \kappa), \forall j\right]$ in (22) is more important than the second one $\left(\rho \leq \bar{\rho}-\lambda_{j} /\left(w^{\prime} \kappa\right), \forall j\right)$, because the latter concerns high number of running trains, and corresponds to the congestion traffic phase of the train dynamics, which is better to avoid (see [13]). We summarize the findings of this section in the following proposition.

Proposition 6: If $\lambda_{j} \leq \min \left(\alpha_{j}, \kappa f_{\max }\right), \forall j$, then there exists a condition on the number $m$ of trains to run on the metro line in such a way that the train dynamics (9) behaves as a max-plus linear one; where the passenger arrivals do not have any effect on the train dynamics. The condition on $m$ is

$$
\frac{L \bar{\lambda}}{v \kappa} \leq m \leq n-\frac{L \bar{\lambda}}{w^{\prime} \kappa}
$$

where $\bar{\lambda}=\max _{j} \lambda_{j}$.

Proof: It follows directly from (18), (19), (21), and (22).

Example 7: With the parameters taken in Table I, we have $f_{\max }=50$ train $/ \mathrm{h}, \kappa=500$ passengers, $v=41.18 \mathrm{~km} / \mathrm{h}$, $w^{\prime}=26.61 \mathrm{~km} / \mathrm{h}$, and $L=17.294 \mathrm{~km}$. Let us notice here that $v_{\text {run }}$ given in Table I corresponds to the free train speed during the intersegment running times without including dwell times, while $v=L / \sum_{j} \underline{t}_{j}$ introduced in [13] corresponds to the free speed during the whole travel time in the line, including train dwell times. The condition $\lambda_{j} \leq \min \left(\alpha_{j}, \kappa f_{\max }\right), \forall j$ under which the max-plus linear dynamics can be retrieved, gives $\bar{\lambda} \leq 6.94$ passengers/s. Under that condition, the number $m$ of trains has to satisfy $3 \bar{\lambda} \leq m \leq 78-4.68 \bar{\lambda}$, in order that the max-plus train dynamics will be attained. For example, we have

1) $\bar{\lambda}=1 \Rightarrow$ no passenger effect with $3 \leq m \leq 73$;

2) $\bar{\lambda}=3 \Rightarrow$ no passenger effect with $9 \leq m \leq 63$;

3) $\bar{\lambda}=5 \Rightarrow$ no passenger effect with $15 \leq m \leq 54$;

4) $\bar{\lambda}=8 \Rightarrow \forall m$ there is a passenger effect, since $\bar{\lambda}>6.94$. Numerical simulations of Section III-D confirm our findings.

\section{Derivation of the Asymptotic Average Dwell Times $w$ and Safe Separation Times $g$ at All Nodes}

From (2), we have $w=t-r$. From (3), we have $t=$ $(m / n) h$. Then, we obtain $w=(m / n) h-r$. Therefore, if we 
TABLE II

Asymptotic Average Train Time-Headway, Frequency, Dwell time at All Nodes and Dwell Time at Platforms; as Functions of the Number of Running Trains. The Average Passenger Arrivals to Platforms Are Given in the Figures (Denoted by $c$ )

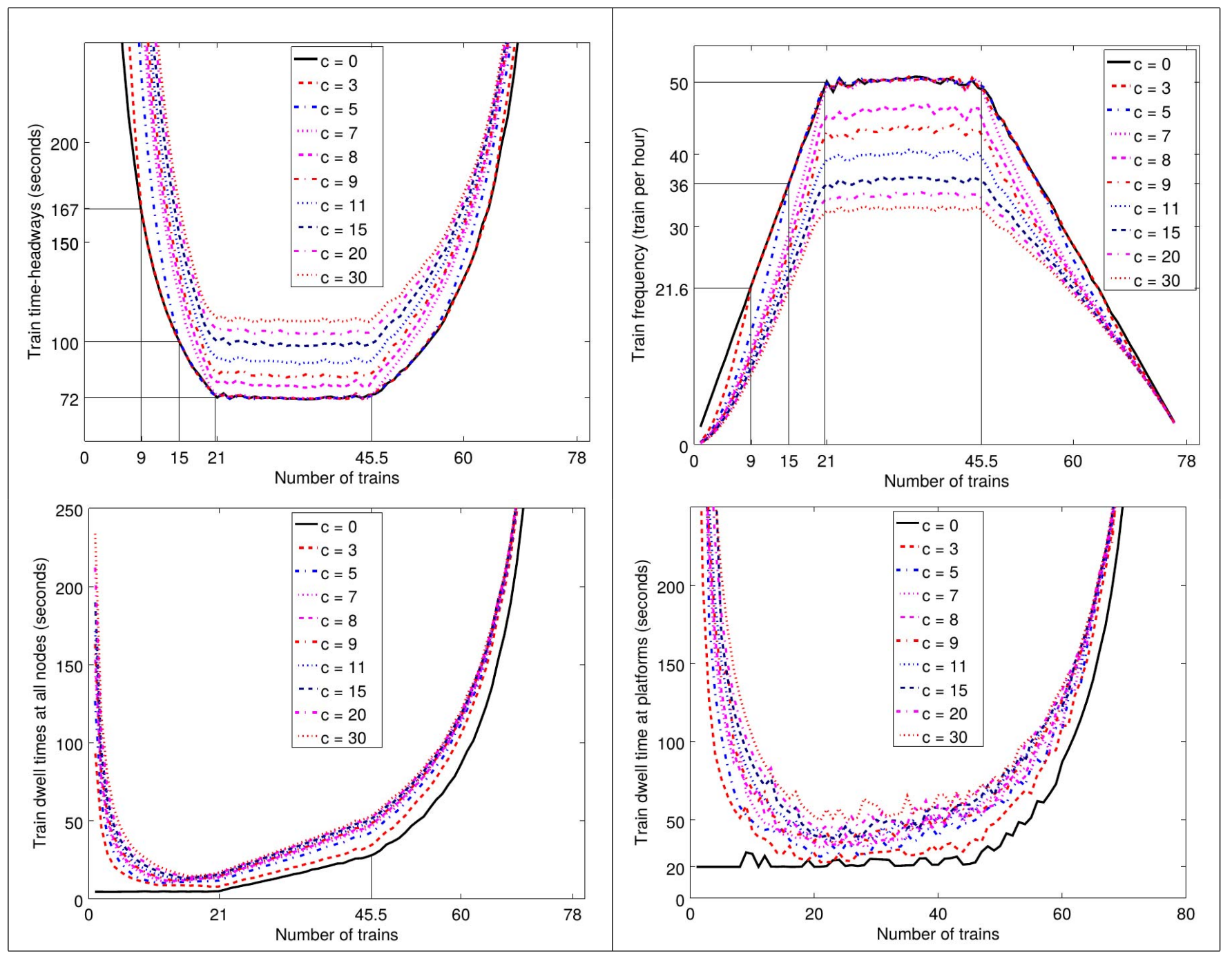

have $h(\rho)$ by simulation [by means of (12)], we deduce $w(\rho)$ as follows:

$$
w(\rho)=(\rho / \bar{\rho}) h(\rho)-r .
$$

Similarly, from (1), we have $g=r+s$. From (3), we have $s=((n-m) / n) h$. Then, we obtain $g=r+((n-m) / n) h$. Therefore, from (12), we obtain

$$
g(\rho)=r+(1-\rho / \bar{\rho}) h(\rho) .
$$

\section{Numerical Results}

In this section, we present some numerical results. We consider the symmetric arrival passenger case where the passenger arrival rates are the same to all the platforms. The rate is then varied in order to derive its effect on the train dynamics and on the physics of traffic. The results are given in Table II. In the top left (respectively, top right) of Table II, we see the increase of the train time-headways (respectively, degradation of the train frequencies) due to increases of the passenger arrival rates. In the second row of Table II, we see the increase of the average dwell times $w$ at all the nodes, and $w^{*}$ at platforms due to increases of the passenger arrival rates.

As shown in Theorem 5, the control law proposed here guarantees train dynamic stability, for every level of passenger demand. For a passenger arrival rate less than or equal to the maximum supply (maximum passenger flow that can be served by the line), the control law guarantees the existence of a number of trains, from which the train frequency is not affected by the passenger demand. We have shown in Example 7 that for a passenger arrival rate equal to 3 (respectively, 5) passengers/s at every platform, we need (at least) 9 (respectively, 15) trains in order that the train frequency coincides with the one obtained by the max-plus dynamics, with no passenger effect. The figures of Table II illustrate this remark. Indeed, we can see on those figures that, starting 
from a number of 9 (respectively, 15) running trains on the metro line, the curves of the asymptotic average time headway $(h)$, frequency $(f)$, and dwell time at all the nodes $(w)$ corresponding to a passenger arrival rate of 3 (respectively, 5) passengers/s [the red (respectively, blue) dashed lines] join the curves of the asymptotic average time headway $(h)$, frequency $(f)$, and dwell time at all the nodes $(w)$ corresponding to a passenger arrival rate of zero passengers (the black continuous line).

For passenger demand levels higher than the maximal supply of the line, the control law guarantees train dynamic stability, but the train frequency is degraded. We have shown in Example 7 that for a passenger arrival rate higher than 6.94 passengers/s, the passenger demand does have an effect on the train frequency whatever the number of trains running on the line. Thus, we can see on Table II that for passenger arrival rates of $8,11,15,20$, and 30 , the curves of the time-headway, frequency, and dwell time at all nodes never join the ones corresponding to rate zero of passenger arrival.

\section{E. Practical Implementability of the Control Law}

Let us first notice that buffer times can be included in our model, in order to resorb eventual disturbances. This can be done, for example, by extending the minimum train dwell times $\underline{w}_{j}$ at platforms, in particular at the level of the two terminal platforms. All the results obtained here remain valid since $\frac{w}{j}$ are parameters of the model. The use of buffer times induces in general train-frequency losses. However, the margins on the train dwell times can be used for a rapid recovery, which enhances robustness.

The control of the train dynamics necessitates a combination of offline calculus and real-time control.

\section{1) Offline Steps:}

a) Consider the max-plus linear dynamics (10) and derive analytically $\tilde{h}(\rho), \forall \rho$ (independent of the travel demand).

b) Deduce $\tilde{\lambda}(\rho), \forall \rho$ by (14) and then the parameters $\bar{w}_{j}(\rho), \forall j, \rho$ by (15) and $\delta_{j}(\rho), \forall j, \rho$ by (16).

c) Simulate the dynamics (9) for every $\rho$ and for varied travel demand level and derive the figures of Table II.

d) For the time period of interest (e.g., peak hour), determine the optimal number of trains to run in order to maximize the train frequency, by means of the figures of Table II.

2) Real-Time Steps (for Fixed $m$ and $\lambda_{j}, \forall j$ ):

a) Retrieve $d_{j}^{k}$ in real time (observation).

b) Iterate (9) with $\bar{w}_{j}$ and $\delta_{j}$ given by (15) and (16).

\section{CONCLUSiON}

We proposed a dynamic programming-based approach for modeling and control of the train dynamics in metro lines. Basing on the conclusions of the max-plus linear model, we proposed an extension to a stochastic dynamic programming model, where the passenger arrivals are taken into account. The models permit the understanding of the effect of the passenger arrivals on the train dwell times at platforms, and by that on the whole dynamics of the trains. The perspectives in this direction of research are numerous. First, the derivation of analytic formulas for the asymptotic average train frequency, dwell time, and safe separation time would bring a better understanding of the traffic control model proposed here. Second, the passenger demand is modeled here through average passenger arrival rates. A dynamic model of the stock of passengers on the platforms would improve the traffic dynamics, particularly by taking into account the capacity limits of the platforms. Another direction of research is to extend the approach to metro lines with junctions (first results have already been obtained in [22]). Finally, other control parameters such as the train running times (speed profiles) can be considered in addition to the train dwell times at platforms (first results have already been obtained in [23]).

\section{REFERENCES}

[1] W. O. Assis and B. E. A. Milani, "Generation of optimal schedules for metro lines using model predictive control," Automatica, vol. 40, no. 8, pp. 1397-1404, 2004.

[2] V. Cacchiani et al., "An overview of recovery models and algorithms for real-time railway rescheduling," Transp. Res. B, Methodol., vol. 63, pp. 15-37, May 2014.

[3] M. G. Crandall and L. Tartar, "Some relations between nonexpansive and order preserving mappings," Proc. Amer. Math. Soc., vol. 78, no. 3 , pp. 385-390, 1980.

[4] J. Cury, F. Gomide, and M. Mendes, "A methodology for generation of optimal schedules for an underground railway system," IEEE Trans. Autom. Control, vol. 25, no. 2, pp. 217-222, Apr. 1980.

[5] O. Engelhardt-Funke and M. Kolonko, "Analysing stability and investments in railway networks using advanced evolutionary algorithms," Int. Trans. Oper. Res., vol. 11, no. 4, pp. 381-394, 2004.

[6] N. Farhi, M. Goursat, and J.-P. Quadrat, "Derivation of the fundamental traffic diagram for two circular roads and a crossing using minplus algebra and Petri net modeling," in Proc. IEEE CDC-ECC, Dec. 2005, pp. 2119-2124.

[7] N. Farhi, M. Goursat, and J. P. Quadrat, "Fundamental traffic diagrams of elementary road networks," in Proc. ECC, Jul. 2007, pp. 4944-4951.

[8] N. Farhi, "Modélisation minplus et commande du trafic de villes Régulières," Ph.D. dissertation, Univ. Paris 1 Panthéon-Sorbonne, Paris, France, 2008.

[9] N. Farhi, "Modeling and control of elementary 2D-traffic systems using Petri nets and minplus algebra," in Proc. IEEE CDC/CCC, Dec. 2009, pp. 2292-2297.

[10] N. Farhi, M. Goursat, and J.-P. Quadrat, "The traffic phases of road networks," Transp. Res. Part C, Emerg. Technol., vol. 19, no. 1, pp. 85-102. 2011.

[11] N. Farhi, "Piecewise linear car-following modeling," Transp. Res. C, Emerg. Technol., vol. 25, pp. 100-112, Dec. 2012.

[12] N. Farhi, C. Nguyen Van Phu, H. Haj-Salem, and J.-P. Lebacque. (2018). "Traffic modeling and real-time control for metro lines." [Online]. Available: https://arxiv.org/abs/1604.04593

[13] N. Farhi, C. Nguyen Van Phu, H. Haj-Salem, and J.-P. Lebacque, "Traffic modeling and real-time control for metro lines. Part I-A max-plus algebra model explaining the traffic phases of the train dynamics," in Proc. ACC, May 2017, pp. 3834-3839.

[14] N. Farhi, C. Nguyen Van Phu, H. Haj-Salem, and J.-P. Lebacque, "Traffic modeling and real-time control for metro lines. Part II-The effect of passenger demand on the traffic phases," in Proc. ACC, May 2017, pp. 3828-3833.

[15] S. Gaubert and J. Gunawardena, "A non-linear hierarchy for discrete event dynamical systems," in Proc. 4th IEE Workshop Discrete Event Syst. (WODES), Cagliari, Italy, 1998.

[16] S. Gaubert and J. Gunawardena, "Existence of eigenvectors for monotone homogeneous functions," HP Labs, Palo Alto, CA, USA, Tech. Rep. HPL-BRIMS-99-08, 1999. 
[17] C. J. Goodman and S. Murata, "Metro traffic regulation from the passenger perspective," Proc. Inst. Mech. Eng. Part F, J. Rail Rapid Transit, vol. 215, no. 2, pp. 137-147, 2001.

[18] J. Gunawardena and M. Keane, "On the existence of cycle times for some non-expansive maps," HP Labs, Palo Alto, CA, USA, Tech. Rep. HPL-BRIMS-95-003, 1995.

[19] IEEE Standard for Communications-Based Train Control (CBTC) Performance and Functional Requirements, IEEE Standard 1474.1-2004, 2004.

[20] W.-S. Lin and J.-W. Sheu, "Metro traffic regulation by adaptive optimal control," IEEE Trans. Intell. Transp. Syst., vol. 12, no. 4, pp. 1064-1073, Dec. 2011.

[21] F. Schanzenbacher, R. Chevrier, and N. Farhi, "Fluidification du trafic transilien: Approche prédictive et optimisation quadratique," in Proc. ROADEF, 2016
[22] F. Schanzenbächer, N. Farhi, Z. Christoforou, F. Leurent, and G. Gabriel, "A discrete event traffic model explaining the traffic phases of the train dynamics in a metro line system with a junction," in Proc. IEEE CDC, Dec. 2017 , pp. $6283-6288$.

[23] F. Schanzenbächer, N. Farhi, F. Leurent, and G. Gabriel, "A discrete event traffic model explaining the traffic phases of the train dynamics on a linear metro line with demand-dependent control," in Proc. Amer. Control Conf., 2018

[24] X. Sun, S. Zhang, H. Dong, and H. Zhu, "Optimal train schedule with headway and passenger flow dynamic models," in Proc. IEEE Int. Conf. Intell. Rail Transp. (ICIRT), Aug./Sep. 2013, pp. $307-312$.

[25] V. V. Breusegem, G. Campion, and G. Bastin, "Traffic modeling and state feedback control for metro lines," in Proc. IEEE Trans. Autom. Control, vol. 36, no. 7, pp. 770-784, Jul. 1991. 